Artigo original

\title{
CONTRIBUICIÓN DE LA CLASE DE EDUCACIÓN FÍSICA AL PROCESO DE ENSEÑANZA-APRENDIZAJE GENERAL DE $1^{\mathrm{er}}$ GRADO.
}

Maristela Bortolon de MATOS $^{1}$

\begin{abstract}
RESUMEN: La investigación realizada constituyo un experimento pedagógico de ler grado (control y experimental), donde se conjugaron contenidos de otras asignaturas (Lengua Portuguesa, Matemática, Ciencias, Estudios Sociales), con de la Educación Física en un mismo proceso, que formaban un todo único en correcta armonía, con el objetivo de reforzar y mejorar los conocimientos adquiridos en esas asignaturas, sin que ello moleste en la consecución de los objetivos esenciales de aquella.Esta investigación se trato precisamente comprobar en la práctica pedagógica que la enseñanza de la Educación Física puede ser efectiva y globalizadora, donde la integración de las disciplinas desarrolle no sólo lo físico, sino lo intelectual, lo afectivo, lo social y otros.
\end{abstract}

Palabras-claves: Educación Física, integración, proceso enseñanza-aprendizaje

Contribuição da disiciplina de educação física ao processo geral de ensinoaprendizagem de 1ạ Série.

RESUMO: A investigação realizada constitui um experimento pedagógico de la Série (controle e exprimento), onde se uniram conteúdos de outras disciplinas (Língua Portuguesa, Matemática, Ciências, Estudos Sociais), com a Educação Física em um mesmo processo, que formavam um todo único em correta harmonia, com o objetivo de reforçar e melhorar os conhecimentos adquiridos nestas disciplinas, sem que estes prejudiquem a execução d'aquelas. Esta investigação buscou comprovar precisamente a prática pedagógica de que o ensino de Educação Física pode ser efetivo e globalizado, aonde a integração das disciplinas não desenvolva somente o corpo mas também o intelectual, o afetivo, o social e outros.

Palavras chaves: Educação Física, integração, Processo Ensino-aprendizagem.

${ }^{1}$ Professora do Instituto Superior de Educação. Boa Vista, Roraima.

Bol. Mus. Integrado de Roraima, Boa Vista 6:31-35. 2000[2007] 


\section{INTRODUCCIÓN}

En la actualidad se repite con frecuencia la necesidad de contribuir, desde cada clase de las diferentes asignaturas, a la fijación de los conocimientos ya impartidos por otros.

En los últimos anos se han realizado señalamientos sobre lo útil e importante que puede ser la Educación Física en el desarrollo del escolar, además de su aporte con su contenido específico. Un de los aspectos señalados como de importancia ha sido la contribución que ella, por razones reconocidas (el gusto que tiene el niño por la Educación Física, el respeto y admiración que siente por el profesor, el placer del niño por la actividad física al aire libre, el sentimiento de libertad de movimientos, etc.), puede aportar a la fijación del conocimiento del escolar durante el proceso docenteeducativo, sin descuidarse del cumplimiento de los objetivos propios de la Educación Física.

Esta investigación se trato precisamente de realizar aportes concretos sobre el tema, consistió en, precisamente, comprobar en la práctica pedagógica que la enseñanza de la Educación Física puede ser efectiva y globalizadora, donde la integración de las disciplinas desarrolle en los niños no sólo lo físico, sino 10 intelectual, lo afectivo, lo social y otros.

La investigación se realizo motivada por la experiencia que surge del trabajo de la autora como profesora de Educación Física durante anos. Tiempo durante el cual aprecio la posibilidad de imbricar los esfuerzos de su clase con las del proceso de las aulas en un fin común, sin deterioro de los objetivos de la actividad y también después de observar, encuestar y analizar la situación de la educación física en las escuelas de Boa Vista, Roraima, Brasil.

\section{DESENVOLVIMIENTO}

El proceso de experimentación en su fase inicial se dividió en dos momentos. En la primera fue hecha una revisión de la documentación en la escuela, como de los planes de clase de Educación Física y de las asignaturas del primer grado de la enseñanza fundamental; la segunda fue la observación de las clases de Educación Física y de las clases teóricas.

La investigación realizada constituyo un experimento pedagógico en condiciones naturales de desarrollo del proceso de enseñanza-aprendizaje de la Educación Física, donde se conjugaron contenidos de otras asignaturas en un mismo proceso, que formaban un todo único en correcta armonía, conforme representación en esquema (anexo).

Se sometieron al experimento dos grupos de niños del primer grado de la enseñanza fundamental. Un grupo constituyó el de control con 23 niños y el otro el experimental con 23 niños, siendo todos de la misma escuela y de composición mixta en cuanto al sexo.

La esencia del experimento consistió en introducir en el grupo experimental, premeditadamente algunos de los contenidos y habilidades de las asignaturas de: Lengua Portuguesa- desarrollar la construcción de textos (historias), reconocer y diferenciar sinónimo y antónimo, femenino y masculino, plural y singular.

Matemática- reconocer y diferenciar par e impar, desarrollar adición y sustracción, ser capaz de diferenciar cantidades (mas, menos e igual).

Ciencias- ser capaz de reconocer las capacidades 
perceptivas (visual, táctil, olfativa, gustativa y auditiva) y conocer la práctica de los hábitos de higiene y posturales.

Estudios Sociales- reconocer el día, mes y el ano, los derechos y deberes (ética y ciudadanía), valorar las fechas conmemorativas. Estos contenidos unidos a los de Educación Física, con el objetivo de reforzar y mejorar los conocimientos adquiridos en esas asignaturas durante las clases de Educación Física, sin que ello moleste en la consecución de los objetivos esenciales de aquella.

La estructura y los aspectos generales seguidos en las clases de Educación Física del grupo experimental fueron:

1-Parte Inicial (15 minutos aproximadamente) - corresponde a la preparación orgánica y psicológica del alumno, para recibir mejor la clase, aclarar las actividades propuestas, iniciando con una actividad agradable y divertida, desarrollándose progresivamente en el esfuerzo y la dificultad, mediante participación activa del alumno.

2-Parte principal (30 minutos)- es destinado a las actividades que contribuyen a la mejor formación corporal del organismo en desarrollo, a través de actividades adecuadas al contingente de edad, en lo tocante a su forma, ritmo, intensidad y desarrollándose en cantidad y calidad conforme los objetivos planificados en Educación Física y de otras disciplinas. Los objetivos que se pretenden alcanzar con este trabajo son representados por el mejor desarrollo físico, mental y social, fortalecimiento general del organismo, desarrollando la elasticidad muscular, flexibilidad articular, coordinación neuromuscular, previniendo y/o realizando corrección de actitudes posturales, utilizando ejercicios estáticos y dinámicos (trabajo muscular).

3-Parte final ( 15 minutos aproximadamente) - Es dedicada en buscar la normalización general del organismo, intentar desarrollar en los alumnos la conciencia del trabajo realizado, reflexionando sobre las actividades desarrolladas, dentro de un ambiente alegre y calmado, enfatizando los contenidos aplicados. Lo cual permitirá una revisión y refuerzo de lo realizado, posibilitando una evaluación general. De esta manera el alumno ira readquiriendo el equilibrio orgánico - emocional necesario para continuar el resto de las clases del día.

4-Evaluación- Los alumnos eran evaluados en el transcurso de la clase, a través del desempeño individual de cada uno, y donde se verificaba la necesidad de atención individual o grupal de refuerzo en el aprendizaje, las actividades de las próximas clases eran planeadas para suplir estas necesidades. Y también en la fase final fue hecha una evaluación general.

5-Consideraciones- En el inicio y fin de las clases de Educación Física los alumnos seguían una organización previamente establecida para dirigir y regresar de la cuadra, para beber agua, utilizar los baños y lavar las manos.

Por este motivo y por otras influencias ajenas, el horario de la fase inicial y final de las clases de Educación Física podría tener variaciones de minutos.

Con el objetivo de comprobar el efecto causado por las clases de Educación Física integrando los contenidos de las demás asignaturas teóricas del grado que cursan, en los niños de primer grado, fueron sometidos a encuesta y entrevista un total de 14 personas que de una u otra forma están ligados al 
proceso docente y desarrollo escolar de estos niños y tuvieron la oportunidad de acompañar todo el proceso del experimento. Ellos fueron- Director, Vice-director, Coordinador pedagógico, Profesores (6), padres (2), agente de salud, Secretaria de la escuela y la alumna de practica docente. Por las respuestas ofrecidas, se considera 100 por ciento a los temas a seguir, se pudo constatar que el planeamiento realizado con las clases del grupo experimental atendió a las necesidades de la disciplina de la Educación Física en todo el periodo; que la enseñanza realizada en las clases de Educación Física fue eficaz; que los medios de enseñanza utilizados permitieron alcanzar los objetivos propuestos, así como los materiales eran adecuados para la actividad a desarrollar; afirmaran que los alumnos se mostraron motivados en el desarrollo de las actividades de la clase. Este fue un factor importante, que a nuestro juicio, contribuyo notablemente a la asimilación de los contenidos en el proceso de integración de las asignaturas. Este factor posibilito la eficacia encontrada por ellos en respuesta anterior, que además propicia los valores también ya señalados. Que en el grupo experimental ocurrió integración, corrobando los resultados que aseveran desarrollo de procesos efectivos $y$ sociales, sin los cuales no lograría con efectividad la integración y cohesión del grupo experimental. Unánimemente hallan importante, la integración de la Educación Física con las asignaturas restantes, que reciben los niños en la escuela y consideran que es positiva totalmente la influencia que este hecho aporta al desarrollo integral del alumno. Todos reconocen que la Educación Física desarrolla valores como respeto, solidaridad y proporciona valoraciones afectivas, cognitivas, sociales y otras, en los niños.
Además de las encuestas y entrevistas aplicadas a las 14 personas, el experimento fue comprobado también a través de entrevistas con los niños del grupo experimental, de las evaluaciones continuas en las clases, tanto de Educación Física cuanto en las clases teóricas, principalmente a través de las clases teóricas, ya que en el principio del experimento el planeamiento de los dos grupos (de control y experimental) era hecho en conjunto por las dos profesoras de clases teóricas, y después de dos meses esto ya no era más posible, pues el grupo experimental estaba bien mas adelantado y por lo tanto ya no se podría planear con los mismos contenidos.

Como se puede observar, los resultados del experimento por las opiniones de estas personas fueron positivos, lográndose los objetivos propuestos durante el proceso. Téngase en cuenta, que entre los encuestados figuraban los profesores de los niños y para nosotros sus criterios fueron fundamentales, no reportándose en conversaciones con ellos alguna opinión negativa en cuanto al proceso y sus resultados.

En fin, consideramos, que de forma general la integración de los contenidos de las asignaturas teóricas con los de Educación Física es sin dudas, muchos de ellos factibles de llevar a cabo y reforzar o mejorar el proceso de aprendizaje, sin perjudicar el logro de los objetivos de la asignatura Educación Física.

\section{CONCLUSIÓN}

Intentando resumir lo acaecido en el experimento, cosa ciertamente dificil por el volumen de actividades realizadas para constatar los resultados, que como se planteaba, no son de naturaleza cuantitativa, por tratarse de dos contingentes de edades muy 
tempranas, que estaban comenzando a leer $y$ escribir $y$ pudiera ser objetiva una evaluación donde ellos sean los principales respondientes a nuestro cuestionamientos. No cabe dudas, que al momento de inicio del experimento ambos grupos presentaban condiciones similares, por el mero hecho que eran alumnos de un mismo nivel y edad, que recibían sus clases con profesoras que impartían un mismo programa, que además ninguno de los dos grupos tenia ventaja, en cuanto a las tareas extra de estudio para mejorar el nivel de aprendizaje.

Las visitas y observaciones a clases y reviso de documentación realizadas, confirman que el solo hecho de cómo recibió las clases el grupo experimental, ya es motivo suficiente para pensar en la superioridad sobre el otro grupo que trabajo de forma tradicional. Por si fuera poco, las encuestas y entrevistas realizadas a los profesionales y familiares y niños mismos del grupo experimental y de control confirman la superioridad del resultado obtenido con este grupo.

Todos concuerdan en que el experimento obtuvo éxito, que ayudó en el aprendizaje de los niños, que el trabajo en conjunto de los profesores hace que el aprendizaje ocurra con mas éxito, que se pueda conocer mejor a los alumnos y con esto atender mejor sus necesidades y especificidad.

En fin, consideramos que el experimento demuestra 1 a posibilidad de aplicación de actividades, tareas, etc., que amplia gama posee la Educación Física, para realizar un trabajo de reforzamiento del conocimiento obtenido por niños en clases teóricas.

\section{BIBLIOGRAFIA}

BARROS, Daisy R. P. e BARROS, Darcymires do Rego. Educação Física na Escola Primária. Rio de Janeiro: Livraria José Olympio Editora, 1972.

BORGES, Célio José. Educação Física Pré-Escolar. Rio de Janeiro:Sprint, 1987 .

BRASIL. Secretaria de Educação Fundamental . Parâmetros Curriculares Nacionais:

Educação Física/Secretaria de Educação Fundamental. -Brasilia: MEC/ SEF, 1997. V.7

BRASIL (1996). Lei de Diretrizes e Bases da Educação Nacional-9394/96 Art.26, parágrafo $3^{a}$. Art.60, parágrafo único.

HILDEBRANDT, Ralf e LAGING, Reiner. Concepções abertas no ensino de Educação Física. Ao Livro Técnico S/A.Rio de Janeiro, 1986.142p.

HURTADO, johann G. G. Melcherts. Educação Física Pré-Escolar e Escolar - $1^{a}$ a $4^{a}$ série: uma abordagem psicomotora. Curitiba: PRODIL. Curitiba, 1987.

HANGEMANN, Marilusi e RODRIGUES, Marta Bergallo. Criança Cresce Brincando: educação física infantil e PréEscola $-1^{a}$ a $4^{a}$ série. Porto Alegre: Magister Ltada, 1991.

PEDAGOGíA. Trabajo colectivo de especialistas del Ministerio de Educación de Cuba bajo la dirección del Instituto Central de Ciencias Pedagógicas. Habana. Editora Pueblo y Educación. 1988. 
